\title{
Isolation and identification of some aerobic bacterial flora from female genitalia in Goats in Babylon city.
}

\author{
Saad Mohsen AL-Sariy \\ 1(Surgery and obstetric department, Veterinary medicine college/Al-Qasim green University, Iraq)
}

\begin{abstract}
The aerobic bacterial flora of the reproductive tracts were investigated using seventeen goats slaughtered at Al-Hilla Medhatiea slaughterhouse. Samples were collected aseptically from the vagina, cervix, uterine body, right and left uterine horns and right and left oviducts and for bacteriological examination. Standard microbiological techniques were used for isolation and identification of bacterial species.

From a total of 119 specimens collected for bacteriological examination from cervix, uterine body, right and left uterine horns, right and left oviducts and vagina (17 each). Bacterial species identified were Staphylococcus aureus (30.25\%) is the highest percentage which were positive for coagulase test, Escherichia coli(26.89\%), Staphylococcus epidermidis and Streptococcus Spp. (10.93\%), Proteus mirabilis. (8.4\%), the pseudomonas aeruginosa $(6.72 \%)$, and finally Listeria monocytogenes (5.88\%), from the positive results, were isolated from all the anatomical sites examined.

In a nutshell, Staphylococcus aureus were dominant in this environment, followed by Escherichia coli.
\end{abstract}

Keywords: Goats, micro flora, Female genital system, Staphylococcus aureus.

\section{Introduction}

The genital tract of animals is known to harbor non-specific bacteria that are sometimes called "normal flora" . Although the type of bacteria present and the role they play is not well understood, under stressful conditions they may cause disease (1). A variety of bacteria have been isolated from the genitalia of the doe and these include (Staphylococci, streptococci , Actinomyces, Pseudomonas, E.coli, mycoplasma and Brucella species (2). , these organisms have been associated with disease condition of the genitalia of animals (3)., other reported that isolation of pathogenic Staphylococci under normal and disease conditions of the genital tract (4).

There are many studies reported the micro flora inhabited in genital system in goats $(5,6,7)$, , recorded that micro flora is usually harmless until presence of predisposing factors such as trauma or another infection which may be pathogenic and cause disease (8)., all these studies and others do not describe the micro flora that found in uterus and oviduct, animals infected with these agents may not be detectable clinically but can usually be identified by laboratory means either in the individual or the flock. E.coli and streptococcus. pyogenes are the most common non-specific infections in goat (9). Thus, the purpose of conducting this study was carried out to determine the aerobic bacteria flora of apparently does from different parts of female genital system.

\section{Materials And Methods}

Collection of samples:

One hundred nineteen samples (vagina, cervix, uterine body, right and left uterine horns and right and left oviducts ) were taken from seventeen goats' genital systems in Al-Hilla Medhatiea slaughterhouse at period from August 2013 to April 2014 and the swabs were rubbed gently on the mucosae from each part of genital system after dissected by surgical blade for bacteriological studies. Each swab was cultured immediately or stored in a transport medium until cultured.

\section{Isolation and identification of bacteria:}

Culture media used for isolation and purification of bacteria included: nutrient agar, blood agar, MacConkey agar, Mannitol salt agar, SS agar medium (for Salmonella) and eosin methylene blue. Inoculated media were incubated aerobically at $37^{\circ} \mathrm{C}$ for 24 hours. All the isolates were stored in brain heart infusion broth with $15 \%$ glycerol at $-20^{\circ} \mathrm{C}$ until further use .

The bacteria isolate were identified their culture, morphology and biochemical characters. For the cultural characteristics, discrete colonies on the agar surface were observed. The shape, size, consistency and color observed. Gram stained slides of the isolate were examined microscopically to study there cellular morphology. The biochemical tests were performed as catalase, oxidase, . IMVIC test (indol production, methyl red, vogas-proskauer and citrate utilization), TSI (triple sugar iron). individually isolated colonies of the same morphology were appropriate agar plate, The culture media prepare depended to routine methods (10). 
The results were shown in Fig. (1) Indicated that growth was observed 69 (57.98\%) were positive, while 50(42.02\%) were negative results from 119 total specimens. These results agree $(9,11)$.

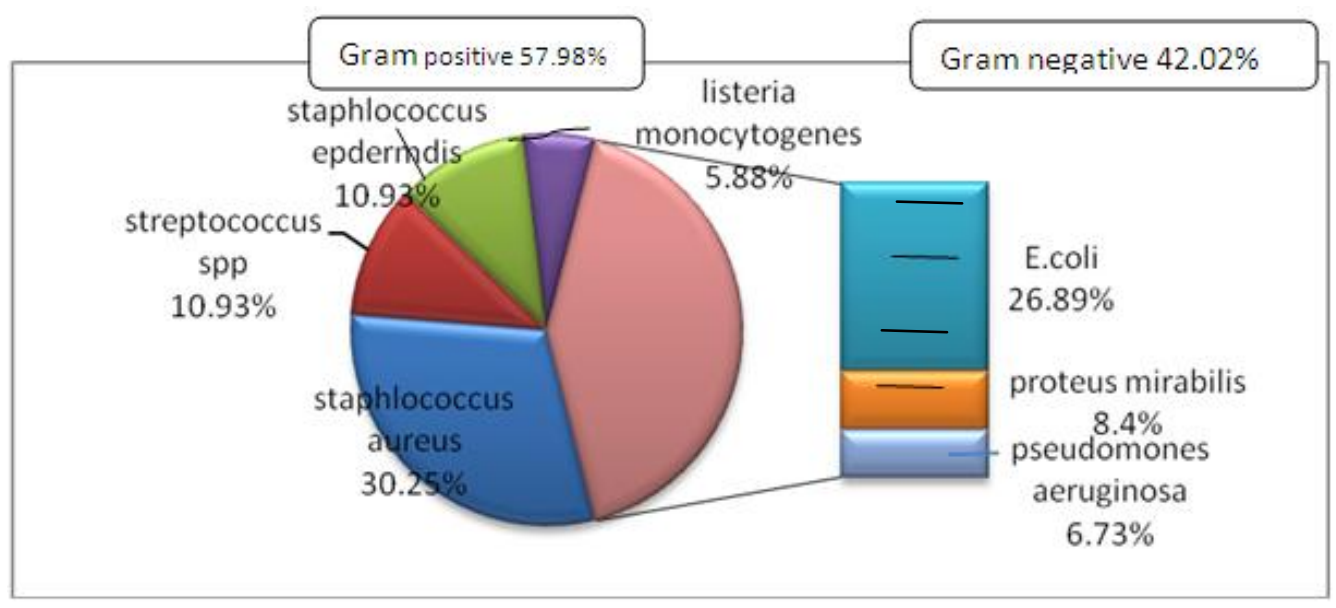

The Laboratory identification of isolates show cultural and staining characteristics of the bacteria isolated from the female genital system in does are presented in table1.The staphylococcus aurous produced gray white or yellow colony on nutrient agar, white to golden colored colony on blood agar. All isolate of staphylococcus aurous were gram positive and arrange cluster and coagulase positive. S.epidermidis were gram positive, growth on blood agar give $\alpha$ hemolysis. Streptococcus spp. produced small, circular and convex colonies on nutrient agar, pin - point colonies surround by clear zones of hemolysis on blood agar. The isolate streptococcus spp. was gram positive and arrange in chains or pairs. Listeria monocytogenes gram-positive coccobacillus which often grows in short chains, motile by peritrichous flagella when grown at $<30^{\circ} \mathrm{C}$ and display a characteristic "tumbling" motility, catalase positive, and indole and oxidase negative, and can hydrolyze aesculin, while E .coli are Gram-negative, , oxidase-negative, bacilli -shaped bacteria lactose fermentation, Pseudomonas aeruginosa are gram-negative, smooth round colonies with a fluorescent greenish color due to the fluorescent pigment pyoverdin , It often produces the nonfluorescent bluish pigment pyocyanin .Proteus spp. are gram-negative, non capsulated, motile rods by swarming motility, Furthermore, these result agree $(12,13)$.

Table 1.Cultural colony characteristics and Grams staining reaction of the bacteria isolated from female genital system in does

\begin{tabular}{|c|c|c|c|c|c|c|c|c|c|c|}
\hline \multicolumn{3}{|c|}{ Staining characters } & \multicolumn{7}{|c|}{ Culture characters } & \multirow{2}{*}{$\begin{array}{l}\text { Identified } \\
\text { organisms }\end{array}$} \\
\hline shape & $\begin{array}{l}\text { Arrange } \\
\text { ment }\end{array}$ & $\begin{array}{l}\text { Gram } \\
\text { stain } \\
\text { reactio } \\
\mathrm{n}\end{array}$ & Nutrient agar & Blood agar & $\begin{array}{l}\text { Mannit } \\
\text { ol salt } \\
\text { agar }\end{array}$ & $\begin{array}{l}\text { maCco } \\
\text { nkey } \\
\text { agar }\end{array}$ & $\begin{array}{l}\text { Eosin } \\
\text { methylen } \\
\text { e blue }\end{array}$ & TSI & IMViC & \\
\hline cocci & clusters & $\begin{array}{l}\text { Gram } \\
+\end{array}$ & $\begin{array}{l}\text { Gray white or } \\
\text { yellowish } \\
\text { colonies }\end{array}$ & $\begin{array}{l}\text { White to } \\
\text { golden } \\
\text {,yellow } \\
\text { colonies B } \\
\text { hemolysis }\end{array}$ & Yellow & $\begin{array}{l}\text { No } \\
\text { growth }\end{array}$ & ---------- & $\begin{array}{l}\mathrm{A} / \mathrm{A} / \\
-/ \text { no } \\
\text { gas }\end{array}$ & -------- & $\begin{array}{l}\text { Staphtococc } \\
\text { us aureus }\end{array}$ \\
\hline cocci & Pairs & $\begin{array}{l}\text { Gram } \\
+\end{array}$ & $\begin{array}{l}\text { White } \\
\text { colonies }\end{array}$ & $\begin{array}{l}\alpha \\
\text { hemolysis }\end{array}$ & $\begin{array}{l}\text { Pink } \\
\text { colonie } \\
\mathrm{S}\end{array}$ & $\begin{array}{l}\text { No } \\
\text { growth }\end{array}$ & ---------- & $\begin{array}{l}----- \\
-\end{array}$ & $\begin{array}{l}------ \\
\end{array}$ & $\begin{array}{l}\text { S.epidermid } \\
\text { is }\end{array}$ \\
\hline $\begin{array}{l}\text { Small } \\
\text { cocci }\end{array}$ & $\begin{array}{l}\text { Chain or } \\
\text { pairs }\end{array}$ & $\begin{array}{l}\text { Gram } \\
+\end{array}$ & $\begin{array}{l}\text { Small, } \\
\text { circular }\end{array}$ & $\begin{array}{l}\text { Pin point } \\
\text { colonies } \\
\text { with } \beta \\
\text { hemolysis }\end{array}$ & $\begin{array}{l}\text { No } \\
\text { growth }\end{array}$ & $\begin{array}{l}\text { No } \\
\text { growth }\end{array}$ & ---------- & $\begin{array}{l}\mathrm{A} / \mathrm{A} / \\
-/ \text { no } \\
\text { gas }\end{array}$ & -------- & $\begin{array}{l}\text { Streptococc } \\
\text { us spp. }\end{array}$ \\
\hline $\begin{array}{l}\text { cocco } \\
\text { bacilli }\end{array}$ & $\begin{array}{l}\text { short } \\
\text { chains, } \\
\text { V shape }\end{array}$ & $\begin{array}{l}\text { Gram } \\
+\end{array}$ & $\begin{array}{l}\text { Motile by } \\
\text { tumbling } \\
\text { movement }\end{array}$ & $\begin{array}{l}\text { beta } \\
\text { hemolytic } \\
\text { colonies, } \\
\text { smooth, } \\
\text { with zones } \\
\text { of hazy } \\
\text { hemolysis }\end{array}$ & $\begin{array}{l}\text { No } \\
\text { growth }\end{array}$ & $\begin{array}{l}\text { No } \\
\text { growth }\end{array}$ & $\begin{array}{l}\text { No } \\
\text { growth }\end{array}$ & $\begin{array}{l}----- \\
---\end{array}$ & -------- & $\begin{array}{l}\text { Listeria } \\
\text { monocytoge } \\
\text { nes }\end{array}$ \\
\hline bacilli & $\begin{array}{l}\text { singly } \\
\text { and in } \\
\text { pairs . }\end{array}$ & Gram - & $\begin{array}{l}\text { circular, with } \\
\text { a convex } \\
\text { elevation, and } \\
\text { a smooth } \\
\text { surface }\end{array}$ & $\begin{array}{l}\text { White } \\
\text { colonies to } \\
\text { brown, } \beta \text { - } \\
\text { hemolysis }\end{array}$ & $\begin{array}{l}\text { No } \\
\text { growth }\end{array}$ & $\begin{array}{l}\text { Pink } \\
\text { colonie } \\
\mathrm{s}, \\
\text { lactose } \\
\text { fremint }\end{array}$ & $\begin{array}{l}\text { Purple } \\
\text { colonies } \\
\text { (lactose } \\
+ \text { ), } \\
\text { metallic } \\
\end{array}$ & $\begin{array}{l}\text { A/A/ } \\
\text {-/gas }\end{array}$ &,,,++-- & $\begin{array}{l}\text { Escherichia } \\
\text { coli }\end{array}$ \\
\hline
\end{tabular}




\begin{tabular}{|c|c|c|c|c|c|c|c|c|c|c|}
\hline & & & & & & ation & sheen & & & \\
\hline $\begin{array}{l}\text { Bacilli } \\
\text { rods }\end{array}$ & $\begin{array}{l}\text { Pairs or } \\
\text { single } \\
\text { Circular, } \\
\text { convex, } \\
\text { and } \\
\text { smooth }\end{array}$ & Gram - & $\begin{array}{l}\text { smooth round } \\
\text { colonies with } \\
\text { produce } \\
\text { pigment and } \\
\text { Motile. }\end{array}$ & $\begin{array}{l}\text { beta- } \\
\text { hemolysis, } \\
\text { the } \\
\text { presence } \\
\text { pigments } \\
\text { and grape } \\
\text { odor. }\end{array}$ & $\begin{array}{l}\text { No } \\
\text { growth }\end{array}$ & $\begin{array}{l}\text { pink/re } \\
\text { d } \\
\text { colorati } \\
\text { on }\end{array}$ & $\begin{array}{l}\text { No } \\
\text { growth }\end{array}$ & $\mathrm{K} / \mathrm{K} /$ &,,,---+ & $\begin{array}{l}\text { Pseudomon } \\
\text { as } \\
\text { aeruginosa }\end{array}$ \\
\hline Rod & $\begin{array}{l}\text { Single } \\
\text { paired } \\
\text { with } \\
\text { flagella }\end{array}$ & Gram _ & $\begin{array}{l}\text { Small } \\
\text { colonies with } \\
\text { swarming } \\
\text { pattern. }\end{array}$ & $\begin{array}{l}\text { Small } \\
\text { colonies }\end{array}$ & $\begin{array}{l}\text { No } \\
\text { growth }\end{array}$ & $\begin{array}{l}\text { Pale } \\
\text { Non- } \\
\text { lactose } \\
\text { fermen } \\
\text { ted }\end{array}$ & $\begin{array}{l}\text { Pink } \\
\text { colonies } \\
\text { (lactose -) }\end{array}$ & $\begin{array}{l}\text { A/A/ } \\
+/ \text { no } \\
\text { gas }\end{array}$ &,,,---+ & $\begin{array}{l}\text { Proteus } \\
\text { mirabilis }\end{array}$ \\
\hline
\end{tabular}

TSI: Triple Sugar Iron , IMViC :I:indole ,M:methyl Red, Vi:Vogus proskuar, C: citrate utilization

The distribution of the bacterial isolates appear from the total 119 samples, each of the specimens isolated from the vagina cervix, uterine body, right and left uterine horns, and right and left oviducts of female genital system in does, the Gram-positive cocci were dominant among the isolates: the frequently isolated species being Staphylococcus aurous (30.25\%), The second dominant bacterium was Escherichia coli (26.89\%), Other species were isolated at relatively lower rates. The bacterial isolates and their absolute/relative abundance are presented on Table 2 and Fig. 2

On the other hand, the bacterial isolates were classified to 7 aerobic bacteria isolated which is Staphylococcus aurous $(30.25 \%)$ is the highest percentage which were positive for coagulase test, Escherichia coli (26.89\%), Staphylococcus epidermidis and Streptococcus Spp. (10.93\%), Proteus mirabilis. (8.4\%), the Pseudomonas aeruginosa $(6.72 \%)$, and finally Listeria monocytogenes(5.88\%), from the positive results , as shown in fig.2. These observations are in accordance with many researchers $(5,9,14$ and 15).

In present study ,These bacteria may be present as saprophytes or (opportunists ) which under conditions of stress may result in disease, the role played by these (non-specific) bacteria is not known but may result in disease under unfavorable conditions of stress(4).

Table 2. - Aerobic bacteria isolated from female genitalia of goats at slaughter.

\begin{tabular}{|c|c|c|c|c|c|c|c|c|}
\hline isolates & $\begin{array}{l}\text { Staphyloco } \\
\text { ccus aureus }\end{array}$ & $\begin{array}{l}\text { Streptococcus } \\
\text { Spp. }\end{array}$ & $\begin{array}{l}\text { Staphylococcus } \\
\text { epidermidis }\end{array}$ & $\begin{array}{l}\text { Listeria } \\
\text { monocytogenes }\end{array}$ & $\begin{array}{l}\text { Escherichia } \\
\text { coli }\end{array}$ & \begin{tabular}{|l|}
$\begin{array}{l}\text { Proteus } \\
\text { mirabilis }\end{array}$ \\
\end{tabular} & $\begin{array}{l}\begin{array}{l}\text { Pseudomonas } \\
\text { aeruginosa }\end{array} \\
\end{array}$ & Total \% \\
\hline $\begin{array}{l}\text { Right } \\
\text { Salpinx }\end{array}$ & $\overline{3}$ & $\overline{3}$ & 4 & 1 & 2 & 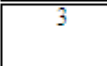 & 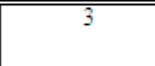 & $19(15.97)$ \\
\hline $\begin{array}{l}\text { Left } \\
\text { Salpinx }\end{array}$ & 5 & 1 & 2 & $\overline{0}$ & $\overline{6}$ & 1 & 3 & $18(15.13)$ \\
\hline $\begin{array}{l}\text { Right } \\
\text { Uterine } \\
\text { horn }\end{array}$ & 8 & 1 & 0 & $\overline{2}$ & 4 & 1 & 0 & $16(13.45)$ \\
\hline $\begin{array}{l}\text { Left } \\
\text { Uterine } \\
\text { horn }\end{array}$ & 6 & 1 & 1 & 1 & 3 & $\overline{0}$ & $\overline{0}$ & $12(10.08)$ \\
\hline $\begin{array}{l}\text { Uterine } \\
\text { body }\end{array}$ & 5 & 2 & 4 & $\overline{0}$ & 3 & 1 & 0 & $15(12.6)$ \\
\hline Cervix & 4 & $\overline{4}$ & 1 & 1 & 7 & 2 & 0 & $19(15.97)$ \\
\hline Vagina & 5 & 1 & 1 & 2 & 7 & 2 & 2 & $20(16.8)$ \\
\hline $\begin{array}{l}\text { Total } \\
\%\end{array}$ & $36(30.25)$ & $13(10.93)$ & $13(10.93)$ & $7(5.88)$ & $32(26.89)$ & $10(8.4)$ & $8(6.72)$ & $1119(10)$ \\
\hline
\end{tabular}




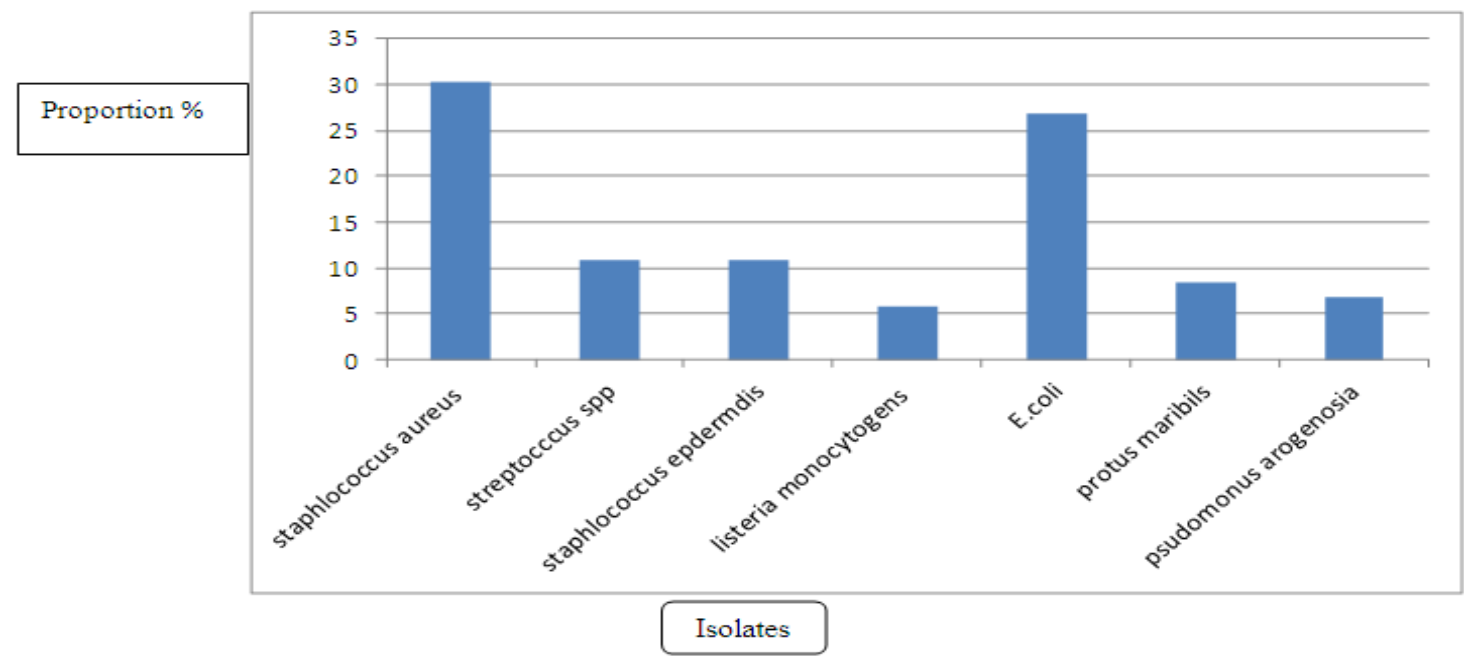

Figure 2. - Relative proportion of different aerobic bacteria isolated from female genital system of goats at slaughter

Further investigations are also required, preferably experimental intervention and examination of specimens collected from pathogenic parts of the female genital system of goats, with the ultimate purpose of clearly verifying the role of bacteria involved in infectious infertility in small ruminants in order to assess their economic impact conclusively.

\section{Conclusions}

From our results, we can concluded that gram positive bacteria particularly Staphylococcus aureus were the most predominant micro flora then E-coli consider gram negative bacteria in normal cases, which under conditions of stress may result in disease .

\section{Acknowledgment}

I wish to thanks veterinarians in Medhatiea slaughterhouse for their efforts and especially Dr. Saleem Najem. I wish to express my heartfelt thanks to Dr. Sulake F.AL-Zubaidi and Dr. Hameedh H. Ajeel for their excellent support and contribution to my research.

\section{References}

[1]. Hirsh,D.C, the genital tract as a microbial habitat, Review of Veterinary Microbiology,1990, 243-244

[2]. Ababneh, A.M and Degefa .T, Bacteriological Findings and Hormonal Profiles in the Post-Partum Balady Goats .Reproductive Domestic .Animal., 4(1), 2007, 12-16.

[3]. Safiriyu,I.O., Waliu,A.S.and Gabriel.E. Exfoliative Vaginal Cytology During the Estrus Cycle of West African Dwarf Goats. Reprod .Nutr.Dev.46, 2006, 87-95

[4]. Bukar Y.M., Amin.J.D., Zaria.L.T., Bacteria flora of the anterior genitalia of the sahelian doe in Maiduguri-borno state , Nigeria., Nigerian Veterinary Journal Vol.28(2), 2007, 60-62.

[5]. N. W. Zaid. Vaginal flora of Iraqi sheep and goats during different reproductive stages. 2009;Al- Anbar J. Vet. Sci., Vol.: 2 No. (1).

[6]. Silva V.F., Damasceno T.E.F., Souza N.J.D., Franco I. \& Costa M.M.Microbiotacérvico-vaginal de ovelhasmestiças e suasusceptibilidadeaosantibióticos, Pesq. Vet. Bras., vol.31, n.7, 2011, 586-590.

[7]. J. Manes, M.A. Fiorentino, G. Kaiser, F. Hozbor, R. Alberio, E. Sanchez, F. Paolicchi Changes in the aerobic vaginal flora after treatment with different intravaginal devices in ewes.2010;Small Rumin. Res(94) 201-204.

[8]. Atwa E, Rady F (2007). Bacteria and fungi associated with abortion in sheep and goat in Menoufiea Governorate. Assiut Vet. Med. J., 53, 326-349.

[9]. Martins, L. T., Neto, P. C. S., Neto, S. G., Rauber, L. P., Bertolini, M., Vieira, A. D. and Mezzalira, A, Microbiological and functional evaluation of an alternative device $(\mathrm{OB}){ }^{\circledR}$ for estrous synchronization in ewes. Cienica- Rural, Santa Maria, 40: (2),2010, 389-395.

[10]. Forbes BA, Sahm DF, and Weissfeld AS. (Bailey and Scotts' Diagnostic microbiology) 12th ed. Elsevier 2007.

[11]. A.F. Majeed, H.M. Al-Rawi, S.M.A. Al-Kubaisi and T.M.N. Al-Jumaily, Vaginal bacteria flora concurred with vaginal sponges in black Iraqi goats, Journal of Veterinary Sciences, Vol. 26, Supplement IV, 2012, 123-124.

[12]. Quinn PJ, Carter ME, Markey B, Carter GR. Clinical veterinary microbiology. An imprint of Elsevier limited; 2004284 - $286 \mathrm{p}$

[13]. Songer, J. G., and Post, K. W. Veterinary Microbiology: Bacterial and Fungal Agents of Animal Disease 1st ed., 434 pp. Elsevier Saunders, St. Louis, MO, 2005. ISBN 0-7216-8717-2.

[14]. El-Arabi A. A., Taylor D. J., Logue D. N. and Benothman M. Isolation and Identification of Bacterial Flora from Reproductive Tracts of Normal Ewes in Glasgow, J Vet Adv., 3(10), 2013, 275-280.

[15]. Sulake Fadhil Al-Zubaidi, Shaimaa O. Hasson, Hameedh H. Ajeel. Isolation and identification of microflora species at different levels of the ewe genital tract. Journal of Agriculture and Veterinary Science 6(3), 2013, 54-57. 